\title{
A new exact solution for boundary layer flow over a stretching plate
}

\author{
Ramesh B. Kudenatti* \\ Department of Mathematics, Bangalore University, Bangalore 560 001, India
}

\section{A R T I C L E I N F O}

\section{Article history:}

Received 12 February 2012

Received in revised form

16 April 2012

Accepted 17 April 2012

Available online 24 April 2012

Keywords:

Boundary layer equations

Pressure gradient

Velocity ratio

Exact solutions

Series solution

\begin{abstract}
A B S T R A C T
In this paper, we give an exact analytical solution of the Falkner-Skan equation for all values of $\beta$. Generalized similarity transformations are used to convert the Prandtl's boundary layer equations into a non-linear ordinary differential equation which accounts two important flow parameters: the pressure gradient parameter $\beta$ and velocity ratio parameter $\epsilon$. Our exact solution method embeds a known closed-form solution for $\beta=-1$ as a special case. We also give the Dirichlet's series solution to the problem for $\epsilon=0$, which is particularly useful when the derivative boundary condition at infinity is zero. We compare the results of both methods with that of direct numerical solution, and found that there is a good agreement between both the results. The results are presented in the form of velocity profiles and skin friction coefficient. Finally, the physical significance of the flow parameters is discussed in detail.
\end{abstract}

(c) 2012 Elsevier Ltd. All rights reserved.

\section{Introduction}

Study of boundary-layer flows of a viscous and incompressible fluid over a continuously stretching surface has significant applications in engineering and industrial processes, such as an aerodynamic extrusion of plastic sheets, liquid film in condensation processes, cooling of a metallic plate in a cooling bath, wire drawing, drawing of plastic films, metal spinning, roofing shingles, insulating materials, cooling of films or sheets, conveyor belts, metallic plates, etc., and in the applications of glass, and polymer industries as well. In all the above applications, the stretching sheet moves with a constant stretching speed and with parallel to its plane. Both the kinematics of stretching sheet and the concurrent heating or cooling during such processes have qualitative influence on the final products. Because of these significant applications, the governing physical problem is converted into an equivalent mathematical one which is necessarily the most celebrated third order non-linear ordinary differential equation: the Falkner-Skan equation which arises in the two-dimensional boundary layer flow of viscous fluid with stream-wise pressure gradient. The Falkner-Skan equation plays a considerable role in the development of boundary layer theory in fluid mechanics. The mathematical treatment of existence and uniqueness results for the Blasius problem, a special case of the Falkner-Skan equation, was established by Weyl [1]. Coppel [2] proved the following important theorem on existence and uniqueness. It states that for all nonnegative $f(0)$ and $f^{\prime}(0)$, the second derivative $f^{\prime \prime}(\eta)$ is positive, zero or negative throughout the interval $0 \leq \eta \leq \infty$ according as $f^{\prime}(0)$ is less than, equal to or greater than 1 . With this restriction on $f^{\prime \prime}(\eta)$, the

\footnotetext{
* Tel.: +91 80 22961424; fax: +9180 22219714 .

E-mail address: ramesh@bub.ernet.in
}

solution is unique. Coppel [2] solved the Falkner-Skan equation for $\beta=-1$ exactly in terms of parabolic cylinder functions. Full numerical solution of the Falkner-Skan equation for all values of $\beta$ was given by Hartree [3], Cebeci and Keller [4] and many more, using the well-known shooting technique. Asaithambi [5] has solved the Falkner-Skan equation numerically using finite difference scheme which is different from shooting technique. In the solution method, an infinite domain was converted into the finite one to achieve the best accuracy.

Because of these significant applications, the Falkner-Skan flow problem has been studied in different contexts. The Falkner-Skan flow in the second grade fluid (Rajagopal et al. [6]), viscoelastic fluid (Olagunju [7]), FENE-P model (Anabtawi and Khuri [8]), heat transfer in nanofluids (Yacob et al. [9]), etc., has been studied.

Due to the high non-linearity of the problem, an exact solution of the Falkner-Skan equation has not been given in the literature for all values $\beta$. It does not admit any exact analytical solution except in very special case. However, Riley and Weidman [10] have given an exact analytical solution of the Falkner-Skan equation for $\beta=-1$ in terms of error and exponential functions which was replica of the solution given by Yang and Chien [11] for different boundary conditions. Most of the solutions are given for $\beta=-1$ for different cases in the literature. Liao [12] developed and implemented successfully a homotopy analysis method for the solution of the FalknerSkan equation for general value of $\beta$. Fang and Zhang [13] have given an exact solution of the Falkner-Skan equation for $\beta=-1$ and explored a different branches of solutions. Recently, Sachdev et al. [14] have found a new way of solving the Falkner-Skan equation for general $\beta$. In their study, exact analytical solution for $\beta=-1$ is obtained in terms of error and exponential functions which is then used to give exact solution for general $\beta$. Kudenatti and Awati [15] have applied the above method, as pioneered by Sachdev et al. [14] 
for the solution of the Falkner-Skan equation for general $\beta$ for a fixed plate and the plate with suction or injection.

Afzal [16] recently has given a new version of the FalknerSkan equation relating free stream velocity to composite reference velocity i.e. sum of the velocities of stretching boundary and free stream. In a boundary layer flow, moving boundary with speed $U_{w}$ is subjected to a free stream speed $U_{\infty}$. For $U_{w}>U_{\infty}$ or $U_{w}<U_{\infty}$, these two problems are physically different which require two sets of boundary layer equations and boundary conditions. Afzal [16] proposed the reference velocity $U(x)$ as $U(x)=U_{w}+U_{\infty}$ which leads to a single set of boundary layer equation along with the boundary conditions, irrespective of whether $U_{w}>U_{\infty}$ or $U_{w}<U_{\infty}$ (see Section 2 for details).

In the present paper, we give an exact analytical solution of the Falkner-Skan equation for all values of $\beta$ using the method pioneered by Sachdev et al. [14]. We solve this problem for general $\beta$ both analytically, in circumstances in which the problem can be solved for the range of $0<\epsilon<\frac{1}{2}$, and numerically for the range $\frac{1}{2}<\epsilon<1$. In the present study, Afzal's [16] work on the solution of the Falkner-Skan equation for $\beta=-1$ has been extended for general values of $\beta$.

The organization of the paper is as follows. The two-dimensional laminar boundary layer equations and similarity transformations are given in Section 2 to derive the Falkner-Skan equation with relevant boundary conditions. However, this derivation can directly be taken from the Afzal [16], but to make this paper self-contained, it is derived in the present paper. An exact solution of the Falkner-Skan equation for $\beta=-1$ is also given in Section 2. Section 3 is devoted to give an exact solution of the Falkner-Skan equation for all general values of $\beta$. In Section 4, we derive another version of the Falkner-Skan equation for $\epsilon=0$. We then apply the Dirichlet series; one of the semi-analytic and highly efficient techniques, for its solution. Section 5 devotes for comparison of the results obtained by our exact and Dirichlet series method with that of direct numerical solution of the Falkner-Skan equation and also with earlier results. The concluding section summarizes the major results of the present study.

\section{Formulation of the problem}

The two-dimensional laminar boundary layer equations for viscous and incompressible fluid subjected to a pressure gradient and stretching of the boundary surface are

$\frac{\partial u}{\partial x}+\frac{\partial v}{\partial y}=0$,

$u \frac{\partial u}{\partial x}+v \frac{\partial u}{\partial y}=-\frac{1}{\rho} \frac{d p}{d x}+v \frac{\partial^{2} u}{\partial y^{2}}$

where $u$ and $v$ are the stream wise and normal velocities in $x$ and $y$ respectively, $\rho$ is the density of the fluid, $p$ is the pressure gradient, $d p / d x=-\rho U_{\infty}\left(d U_{\infty} / d x\right), U_{\infty}$ is the velocity at the edge of the boundary layer of thickness $\delta$, and $v$ is the kinematic viscosity. The relevant boundary conditions are given as follows:

$u=U_{w}(x), \quad v=0$ at $y=0 ; \quad u=U_{\infty}(x)$ as $\frac{y}{\delta} \rightarrow \infty$,

where $U_{w}(x)$ is the velocity of the stretching surface. Using similarity transformations as in Afzal [16] in the above systems (1)-(3), we get

$f^{\prime \prime \prime}(\eta)+f(\eta) f^{\prime \prime}(\eta)+\beta\left(\epsilon^{2}-f^{\prime 2}(\eta)\right)=0, \quad,=\frac{d}{d \eta}$, with the boundary conditions

$f(0)=0, \quad f^{\prime}(0)=1-\epsilon, \quad f^{\prime}(+\infty)=\epsilon$,

where $\epsilon=U_{\infty} /\left(U_{\infty}+U_{w}\right)$ and $\beta=2 m /(1+m)$. For $\epsilon=1$, Eqs. (4) and (5) reduce to the classical Falkner-Skan equation and for $\epsilon<0$ and $\epsilon>1$, both the free stream and the wall are moving in negative $x$-direction. For $0<\epsilon<1$ both the wall and the free stream are moving in positive $x$-direction. The Falkner-Skan equation is one of the most celebrated boundary layer equations which describes the viscous two-dimensional laminar boundary layer flow over a semiinfinite flat plate under stream-wise pressure gradient. The FalknerSkan equation includes many special cases such as a flat plate boundary layer flow when $\beta=0$ (the Blasius flow), the case $\beta>0$ corresponds to a favorable external pressure gradient in the stream direction, for $\beta<0$, the flow has an adverse pressure gradient, the stagnation flow towards a flat plate when $\beta=1$. Although, the mathematical equation for two cases $\epsilon=0$ and $\epsilon=1$ looks similar but their physical behavior is entirely different which is obvious from the boundary conditions. In the following section, we give an exact analytical solution of systems (4) and (5) for general $\beta$ in the range $0<\epsilon<\frac{1}{2}$.

\subsection{The exact solution for $\beta=-1$}

Twice integration of systems (4) and (5) for $\beta=-1$ with respect to $\eta$ gives the Riccati equation of the type

$f^{\prime}(\eta)+\frac{f^{2}(\eta)}{2}=\frac{\epsilon^{2}}{2} \eta^{2}+\delta \eta+1-\epsilon$.

Solution of Eq. (6) is

$$
f(\eta)=\epsilon \eta+\frac{\delta}{\epsilon}-\frac{\frac{\delta}{\epsilon} \exp \left(-\frac{\left(\delta \eta+\frac{\epsilon^{2} \eta^{2}}{2}\right)}{\epsilon}\right)}{1-\sqrt{2 \pi \epsilon} \frac{\delta}{4 \epsilon^{2}} \exp \left(\frac{\delta^{2}}{2 \epsilon^{3}}\right)\left(\operatorname{erf}\left(\frac{\epsilon \eta+\frac{\delta}{\epsilon}}{\sqrt{2 \epsilon}}\right)-\operatorname{erf}\left(\frac{\delta}{\epsilon \sqrt{2 \epsilon}}\right)\right)},
$$

provided $\delta^{2}=2 \epsilon^{2}(1-2 \epsilon)$ and $\operatorname{erf}(Z)=(2 / \sqrt{\pi}) \int_{0}^{Z} e^{-t^{2}} d t$. It is important to note that the above exact analytical solution (7) is the correct one as the same solution that is obtained by Afzal [16] has wrongly typed or given (see Eq. (11) of that paper).

The axial velocity gradient at the wall is given by Eq. (7)

$f^{\prime \prime}(0)=\delta= \pm 2 \epsilon \sqrt{\frac{1}{2}-\epsilon}$.

Eq. (8) shows that there are two solutions for $\epsilon<\frac{1}{2}$, and solution does not exist for $\epsilon>\frac{1}{2}$. It may be observed that $f(\eta)=\eta / 2$ for $\epsilon=\frac{1}{2}$ is the exact solution of (4) and (5). This is a critical solution which changes the analytic character of the solution across the parametric value $\epsilon=\frac{1}{2}$.

This paper devices a method that helps in obtaining a new exact analytical solution of the Falkner-Skan equations (4) and (5) for general values of $\beta$. In the following section this has been achieved using the method pioneered by Sachdev et al. [14].

\section{The exact solution for general $\beta$}

Observe that we have obtained a closed form solution (7) of the Falkner-Skan equations (4) and (5) for $\beta=-1$ which can be rewritten as

$f(\eta)=\epsilon \eta+\frac{\delta}{\epsilon}-\frac{\frac{\delta}{\epsilon}}{\left(\exp \left(\frac{-\delta^{2}}{2 \epsilon^{3}}\right)+\sqrt{2 \pi \epsilon} \frac{\delta}{4 \epsilon^{2}} \operatorname{erf}\left(\frac{\delta}{\epsilon \sqrt{2 \epsilon}}\right)\right) \exp \left(\frac{X^{2}}{2 \epsilon}\right)-\sqrt{2 \pi \epsilon} \frac{\delta}{4 \epsilon^{2}} \exp \left(\frac{X^{2}}{2 \epsilon}\right) \operatorname{erf}\left(\frac{X}{\sqrt{2 \epsilon}}\right)}$, 
where $X=\epsilon \eta+\delta / \epsilon$. This, for convenience, can be rewritten again as

$f(\eta)=\epsilon \eta+\frac{\delta}{\epsilon}-\frac{\delta}{\epsilon G(\eta)}$,

for general values of $\beta$. The function $G(\eta)$ easily generalizes the solution of the Falkner-Skan equation for general $\beta$. Substituting (10) into (4), we get the following equation for $G(\eta)$ :

$$
\begin{gathered}
\epsilon^{2} G^{2} G^{\prime \prime \prime}-G\left[6 \epsilon^{2} G^{\prime}+\delta-\left(\epsilon \eta+\frac{\delta}{\epsilon}\right) G\right] G^{\prime \prime}+6 \epsilon^{2} G^{3} \\
+\left[\delta(2-\beta)-2 \epsilon\left(\epsilon \eta+\frac{\delta}{\epsilon}\right) G\right] G^{\prime 2}-2 \beta \epsilon G^{2} G^{\prime}=0,
\end{gathered}
$$

and the boundary conditions (5) become

$G(0)=1, \quad G^{\prime}(0)=\frac{\delta}{2 \epsilon^{2}}, \quad G(+\infty)=\infty$,

where $G=G(\eta)$. It readily follows from (10) that a closed form solution of (11) and (12) for $\beta=-1$ is given by

$$
\begin{aligned}
G(\eta)= & \left(\exp \left(\frac{-\delta^{2}}{2 \epsilon^{3}}\right)+\sqrt{2 \pi \epsilon} \frac{\delta}{4 \epsilon^{2}} \operatorname{erf}\left(\frac{\delta}{\epsilon \sqrt{2 \epsilon}}\right)\right) \exp \left(\frac{X^{2}}{2 \epsilon}\right) \\
& -\sqrt{2 \pi \epsilon} \frac{\delta}{4 \epsilon^{2}} \exp \left(\frac{X^{2}}{2 \epsilon}\right) \operatorname{erf}\left(\frac{X}{\sqrt{2 \epsilon}}\right) .
\end{aligned}
$$

The error and exponential functions in the above expression are entire functions. As these functions may be expanded in Taylor series about $\eta=0$ which have an infinite radius of convergence, we expect the similar analysis for other values $\beta$. This motivates us to write the series representation as

$G(\eta)=\sum_{n=0}^{\infty} a_{n} \eta^{n}$

for general $\beta$. Substituting the series (14) into (11) and equating the like powers of $\eta$ to zero, we get

$$
a_{0}=1, \quad a_{1}=\frac{\delta}{2 \epsilon^{2}}, \quad a_{3}=\frac{\delta\left(4 \beta \epsilon^{5}+\delta^{2}(-3+\beta \epsilon)+24 \epsilon^{4} a_{2}\right)}{24 \epsilon^{6}},
$$$$
a_{4}=\frac{\delta^{2}\left(2(1+6 \beta) \epsilon^{5}+\delta^{2}(-6+\epsilon+2 \beta \epsilon)\right)+4 \epsilon^{4}\left(2(-1+2 \beta) \epsilon^{5}+\delta^{2}(6-\epsilon+2 \beta \epsilon)\right) a_{2}+96 \epsilon^{8} a_{2}^{2}}{96 \epsilon^{8}},
$$$$
a_{5}=\frac{1}{480 \epsilon^{9}} \delta\left(8(-1+\beta) \beta \epsilon^{9}+2 \delta^{2} \epsilon^{4}\left(5-3 \beta \epsilon+3 \beta^{2} \epsilon\right)\right.
$$$$
+\delta^{4}\left(4+\beta^{2} \epsilon-\beta(3+\epsilon)\right)+4 \epsilon^{3}\left(10(-1+6 \beta) \epsilon^{5}\right.
$$$$
\left.\left.+\delta^{2}(-30-3 \epsilon+16 \beta \epsilon)\right) a_{2}+16 \epsilon^{7}(45+(-1+2 \beta) \epsilon) a_{2}^{2}\right),
$$

$$
a_{6}=\frac{1}{5760 \epsilon^{12}}\left(\delta ^ { 2 } \left(4\left(-3-16 \beta+60 \beta^{2}\right) \epsilon^{10}+3 \delta^{4}(30+\epsilon-22 \beta \epsilon\right.\right.
$$$$
\left.+\left(-1+4 \beta^{2}\right) \epsilon^{2}\right)+2 \delta^{2} \epsilon^{5}\left(15-6 \epsilon+56 \beta^{2} \epsilon\right.
$$$$
-6 \beta(25+2 \epsilon)))+4 \epsilon^{4}\left(4\left(3-8 \beta+4 \beta^{2}\right) \epsilon^{10}\right.
$$$$
+\delta^{4}\left(-360+3(13+34 \beta) \epsilon+\left(3-12 \beta+10 \beta^{2}\right) \epsilon^{2}\right)
$$$$
\left.+2 \delta^{2} \epsilon^{5}\left(15+6 \epsilon+20 \beta^{2} \epsilon-6 \beta(-55+4 \epsilon)\right)\right) a_{2}
$$$$
\left.+96 \epsilon^{8}\left(10(-1+2 \beta) \epsilon^{5}+\delta^{2}(45-7 \epsilon+14 \beta \epsilon)\right) a_{2}^{2}+5760 \epsilon^{12} a_{2}^{3}\right),
$$

and the general recurrence relation to obtain the remaining coefficients

$$
\begin{aligned}
a_{n+3}= & \frac{\epsilon}{\delta(1+n)(2+n)(3+n)} \\
& \times\left(\sum _ { k = 0 } ^ { n } \left(\frac { \delta ^ { 2 } } { \epsilon ^ { 2 } } ( k + 1 ) \left((\beta-2)(n-k+1) a_{k+1} a_{n-k+1}\right.\right.\right.
\end{aligned}
$$

$$
\begin{aligned}
& \left.+(k+2) a_{k+2} a_{n-k}\right)+\sum_{m=0}^{k}((m+1)(k+1-m) \\
& \times\left(\frac{6 \delta}{\epsilon}(k-m+2) a_{k-m+2} a_{n-k}-\frac{6 \delta}{\epsilon}(n-k+1) a_{k+1-m} a_{n+1-k}\right. \\
& \left.\left.+\frac{2 \delta^{2}}{\epsilon^{2}} a_{k+1-m} a_{n-k}\right) a_{m+1}\right) \\
& \left.+\sum_{m=0}^{n-k}\left((k+1)\left(2 \beta \delta a_{k+1}-\frac{\delta^{2}}{\epsilon^{2}}(k+2) a_{k+2}\right) a_{m} a_{n-k-m}\right)\right) \\
& +\sum_{k=0}^{n-1}\left(\sum_{m=0}^{k} 2 \delta(m+1)(k+1-m) a_{m+1} a_{k+1-m} a_{n-1-k}\right. \\
& -\frac{\delta}{\epsilon} \sum_{m=0}^{n-k}(k+1)(k+2)(k+3) a_{m} a_{k+3} a_{n-k-m} \\
& \left.\left.-\sum_{m=0}^{n-k-1} \delta(k+1)(k+2) a_{m} a_{k+2} a_{n-k-m-1}\right)\right) \text { for } n=1,2,3, \ldots
\end{aligned}
$$

Notice from (16) that all the coefficients $a_{n}$ have been obtained in terms of the unknown $a_{2}$. This unknown $a_{2}$ characterizes the coefficient of skin friction, must be obtained such that the derivative condition at $\infty$ is satisfied (see the last boundary condition in (5)). This is equivalent to determine the value of either $a_{2}$ of the series (14) or $f^{\prime \prime}(0)$ of systems (4) and (5) because these are intrinsically related to each other through Eq. (10):

$a_{2}=\left(f^{\prime \prime}(0)+\frac{\delta^{3}}{2 \epsilon^{3}}\right) / 2 \epsilon \delta$.

The coefficients $a_{n}$ involve two arbitrary constants, namely, $\delta$ and $f^{\prime \prime}(0)$. To determine one of the unknown constants $a_{2}$ or $f^{\prime \prime}(0)$ and $\delta$, we match the series expansion of the closed form solution (13) with that of series (14) with $\beta=-1$, which gives the same constant as $\delta=\epsilon \sqrt{2(1-2 \epsilon)}$. The other constant $a_{2}$ or $f^{\prime \prime}(0)$ remains to be determined. To determine the constant $f^{\prime \prime}(0)$, integrate (4) from $\eta=0$ to $\eta=\infty$ and using the conditions (5), we get

$\int_{0}^{\infty}\left(f^{\prime}(\eta)-f^{\prime 2}(\eta)\right) d \eta+\beta \int_{0}^{\infty}\left(\epsilon^{2}-f^{\prime 2}(\eta)\right) d \eta=f^{\prime \prime}(0)$.

Since, left hand side of (18) also involves $f^{\prime \prime}(0)$, it can be solved iteratively. Our results for $f^{\prime \prime}(0)$ thus obtained agrees well with that obtained by numerical solution of Falkner-Skan equations (4) and (5) for all values of $\beta$. However, in order to effectively illustrate the method used to find $f^{\prime \prime}(0)$, we rewrite the above integral relation (18) as

$\int_{0}^{\eta_{\max }}\left(f^{\prime}(\eta)-f^{\prime 2}(\eta)\right) d \eta+\beta \int_{0}^{\eta_{\max }}\left(\epsilon^{2}-f^{\prime 2}(\eta)\right) d \eta=f^{\prime \prime}(0)$,

where $\eta_{\max }=\lim \eta \rightarrow \infty$. Since, $f^{\prime \prime}(0)$ appears on both sides of the above relation, it has to be solved iteratively for all involved parameters. The solution of the above asymptotic integral relation is too complicated by the fact that the boundary condition is specified at infinity. In the integrations, infinity is numerically approximated by the large value of independent variable (i.e. $\eta_{\max }$ ). There is no such priori general method for estimating this value. Selecting too small value for $\eta_{\max }$ does not assure convergence to the required accuracy. And also selecting too large value for $\eta_{\max }$ results either in asymptotic divergence series or in slow convergence of the series to satisfy derivative boundary condition at infinity. Hence, a method must be 
a

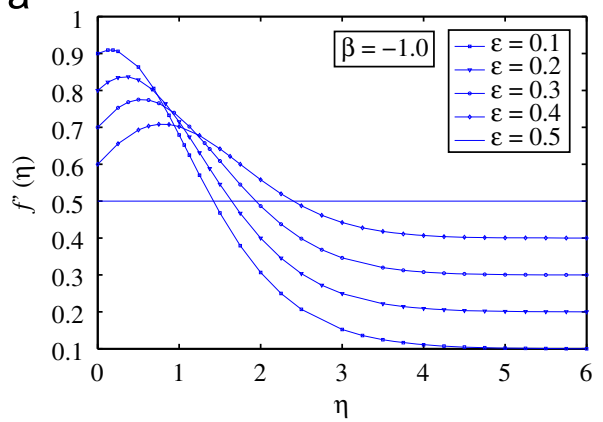

C

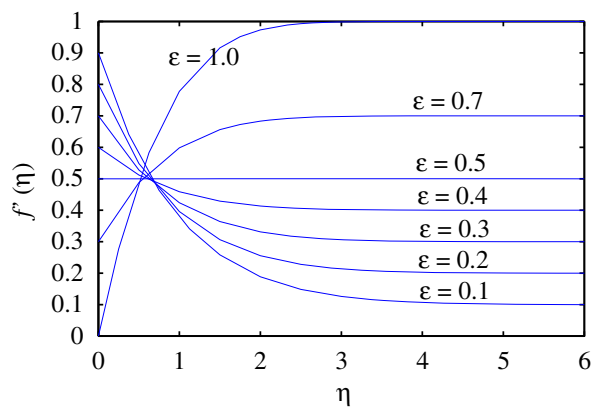

b

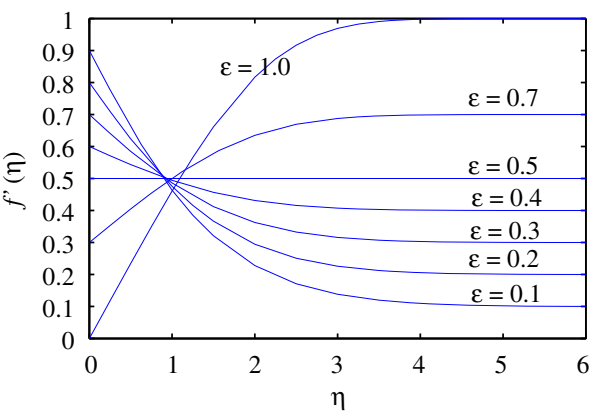

d

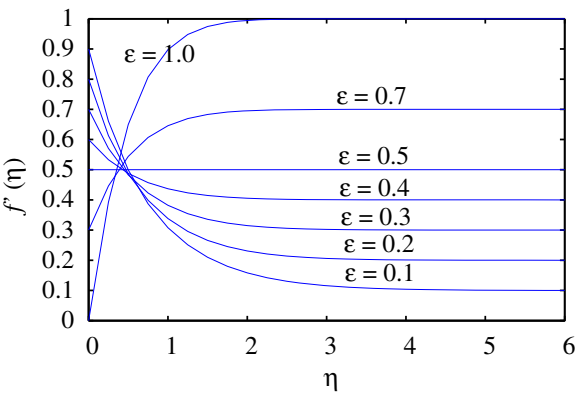

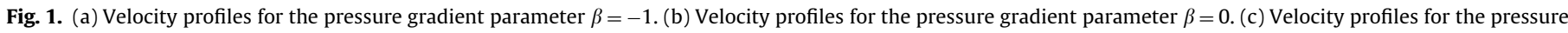
gradient parameter $\beta=1.0$. (d) Velocity profiles for the pressure gradient parameter $\beta=2.5$.

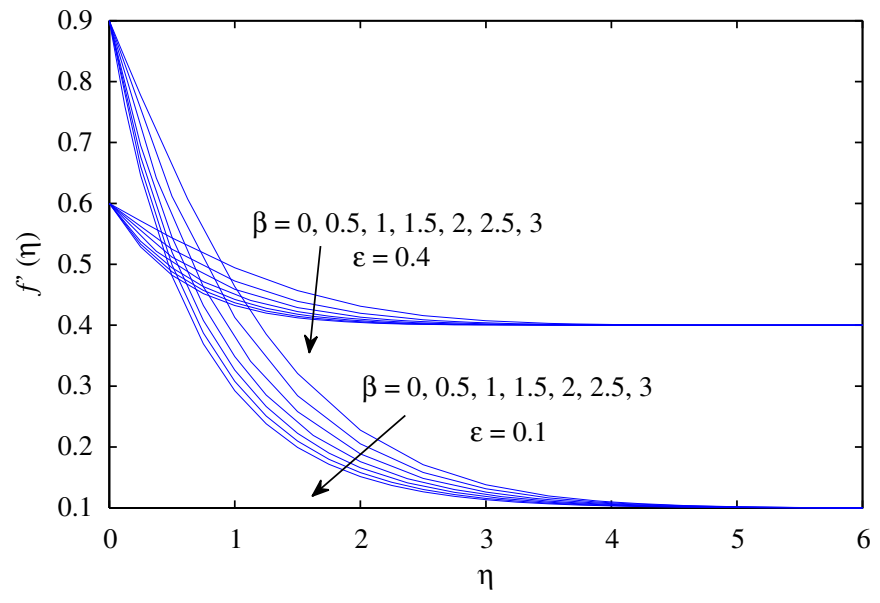

Fig. 2. Velocity profiles for different sets of pressure gradient parameter $\beta$ and velocity ratio parameter $\epsilon$.

devised to logically estimate the value of $\eta_{\max }$ to get $f^{\prime \prime}(0)$ up to required accuracy. In the integration, the initial approximation of $f^{\prime \prime}(0)=0.126491$ is chosen from exact analytical solution for $\beta=-1$ and $\epsilon=0.1$ for all other parameter $\beta$ and $\epsilon$ to ensure the fast convergence. For small value of $\eta_{\max }$, the series is well behaved and can be integrated. So, in the process, once $f^{\prime \prime}(0)$ is assumed and the Pade's approximants which extend the region of validity of convergence, are used, the integral relation can be integrated without any difficulty to determine a nearly correct value of $f^{\prime \prime}(0)$. With fewer iterations, $f^{\prime \prime}(0)$ can be obtained up to any desired accuracy.

The results obtained by the method described above have been used to plot Figs. 1(a)-(d) and 2 and in Table 1.

\section{Solution for $\epsilon=0$}

Flows in the continuously stretching surface have been studied in the past due their industrial applications. Since, the surface is
Table 1

Comparison of skin friction coefficient $f^{\prime \prime}(0)$ obtained by our method (Eqs. (11)-(15)) with that of direct numerical solution.

\begin{tabular}{|c|c|c|c|}
\hline$\beta$ & $\epsilon$ & $\begin{array}{l}\text { Present method } \\
\text { (Eqs. (11) and (15)) }\end{array}$ & $\begin{array}{l}\text { Numerical } \\
\text { method }\end{array}$ \\
\hline \multirow[t]{5}{*}{-1.0} & 0.1 & 0.126491 & 0.126496 \\
\hline & 0.2 & 0.219089 & 0.219090 \\
\hline & 0.3 & 0.268328 & 0.268327 \\
\hline & 0.4 & 0.252982 & 0.252982 \\
\hline & 0.5 & 0.0 & 0.0 \\
\hline \multirow[t]{5}{*}{0.0} & 0.1 & -0.492625 & -0.494005 \\
\hline & 0.2 & -0.363901 & -0.363420 \\
\hline & 0.3 & -0.237219 & -0.237159 \\
\hline & 0.4 & -0.115811 & -0.115816 \\
\hline & 0.5 & 0.0 & 0.0 \\
\hline \multirow[t]{5}{*}{0.5} & 0.1 & -0.675918 & -0.675009 \\
\hline & 0.2 & -0.513980 & -0.513391 \\
\hline & 0.3 & -0.346194 & -0.346629 \\
\hline & 0.4 & -0.175403 & -0.175339 \\
\hline & 0.5 & 0.0 & 0.0 \\
\hline \multirow[t]{5}{*}{1.0} & 0.1 & -0.823981 & -0.823675 \\
\hline & 0.2 & -0.633671 & -0.633832 \\
\hline & 0.3 & -0.432951 & -0.432614 \\
\hline & 0.4 & -0.221982 & -0.221089 \\
\hline & 0.5 & 0.0 & 0.0 \\
\hline \multirow[t]{5}{*}{1.5} & 0.1 & -0.951872 & -0.951911 \\
\hline & 0.2 & -0.736812 & -0.736547 \\
\hline & 0.3 & -0.505918 & -0.505225 \\
\hline & 0.4 & -0.259021 & -0.259374 \\
\hline & 0.5 & 0.0 & 0.0 \\
\hline \multirow[t]{5}{*}{2.5} & 0.1 & -1.169812 & -1.169574 \\
\hline & 0.2 & -0.909281 & -0.909461 \\
\hline & 0.3 & -0.626233 & -0.626627 \\
\hline & 0.4 & -0.323034 & -0.323012 \\
\hline & 0.5 & 0.0 & 0.0 \\
\hline
\end{tabular}

flexible, the filament may be stretched during the course of ejection and so only the surface velocity deviates without being uniform. This problem has been extensively studied since Sakiadis 
[17] investigated the boundary layer flow on a continuous solid surface with constant speeds. Numerous papers are available on stretching sheet problems under different contexts, for example, Crane [18], Zaturska and Banks [19], Guedda [20], Bataller [21], Bognar [22] and references therein. Most of these analyses have been given by numerical methods such as $\mathrm{R}-\mathrm{K}$ methods along with shooting algorithm, Keller-box method, finite difference method, etc. Boundary layer equations in the stretching sheet do not, in general, admit any closed-form solution for involved parameter except in a very-special-case because of their high non-linearity, and also, the infinite domain demands special mathematical tools for further analysis. Therefore, we present below a elegant method called the Dirichlet series (or exponential series ) for the solution of the boundary layer flow problems.

The Dirichlet series solution method ideally suits for specific type of boundary condition where derivative condition at far distance is zero. An advantage of this method is that it helps in obtaining the derived quantities such as velocity profiles $\left(f^{\prime}(\eta)\right)$ and skin friction $\left(f^{\prime \prime}(0)\right)$, directly compared to pure numerical methods. Because of these significant advantages, Kravnchenko and Yablonskii [23] were the first to use the Dirichlet series for solving third order non-linear boundary value problem over infinite domain. A general discussion of the convergence of the Dirichlet series may be found in Riesz [24].

For $\epsilon \rightarrow 0$, the Falkner-Skan equations (4) and (5) take the form of

$f^{\prime \prime \prime}(\eta)+f(\eta) f^{\prime \prime}(\eta)-\beta f^{\prime 2}(\eta)=0$,

and the boundary conditions

$f(0)=0, \quad f^{\prime}(0)=1, \quad f^{\prime}(+\infty)=0$.

We seek Dirichlet series solution for Eq. (20) in the form

$f(\eta)=b_{0}\left(1+\sum_{n=1}^{\infty} b_{n} a^{n} e^{-n b_{0} \eta}\right)$

where the constants $b_{0}>1$ and $a$ are to be determined. Note that the above base function automatically satisfies the derivative boundary condition at infinity. Substituting this base function (22) in systems (20) and (21), we get the following recurrence relation:

$$
\begin{gathered}
-\sum_{n=1}^{\infty} n^{3} b_{n} a^{n} e^{-n b_{0} \eta}+\sum_{n=1}^{\infty} n^{2} b_{n} a^{n} e^{-n b_{0} \eta}+\sum_{n=2}^{\infty} \sum_{k=1}^{\infty} k^{2} b_{k} b_{n-k} a^{n} e^{-n b_{0} \eta} \\
-\beta \sum_{n=2}^{\infty} \sum_{k=1}^{\infty} k(n-k) b_{k} b_{n-k} a^{n} e^{-n b_{0} \eta}=0
\end{gathered}
$$

to obtain the coefficients $b_{n}$ as a function of unknown constants $b_{0}$ and $a$. Note, however, that for $n=1$, the above recurrence relation satisfies the relation: $-b_{1} a+b_{1} a=0$. Therefore, we rewrite the above recurrence relation (23) as

$b_{n}=\frac{1}{n^{2}(n-1)} \sum_{k=2}^{n-1}\left(k^{2}-\beta k(n-k)\right) b_{k} b_{n-k}$,

for $n=2,3,4, \ldots$. We know that the radius of convergence of the Dirichlet series can be obtained, and our computations show that, if $\left|b_{1}\right|<1$, then the series converges absolutely for $\eta>$ $-\left(\ln \left(\lim \left|b_{n} / b_{n+1}\right|\right)-\ln |a|\right)$. Detailed convergence criterion of the above series can be found in Riesz [24].

It is important to obtain the shear stress at the surface $f^{\prime \prime}(0)$ as

$f^{\prime \prime}(0)=b_{0}^{3} \sum_{n=1}^{\infty} n^{2} b_{n} a^{n}$

However, as noted earlier, the series (22), as it is, contains two free-parameters $b_{0}$ and $a$ which are yet to be determined. To determine these constants, we make use of other two initial conditions in (21) namely

$$
\begin{aligned}
& f(0)=b_{0}\left(1+\sum_{n=1}^{\infty} b_{n} a^{n}\right)=0, \\
& f^{\prime}(0)=-b_{0}^{2} \sum_{n=1}^{\infty} n b_{n} a^{n}=1 .
\end{aligned}
$$

We use Newton's method for non-linear equations to determine these unknown parameters up to required accuracy for all values of $\beta$. The results thus obtained for various values of $\beta$ by the present Dirichlet series solution are seen to agree with those produced by the numerical solution of the boundary layer systems (20) and (21) and are presented in Table 2. From this table, the skin friction value $f^{\prime \prime}(0)$ compares well with that of numerical solution for all values of $\beta$. This explicates the advantages of the method over pure numerical methods. Once the constants $a$ and $b_{0}$ are determined, it is important to investigate the velocity behavior. We plot the variation of velocity profiles $f^{\prime}(\eta)$ for different values of $\beta$ which are shown in Fig. 3.

Note that systems (20) and (21) admit an analytical solution for $\beta=1$

$f(\eta)=1-e^{-\eta}$,

and for $\beta=-1$

$f(\eta)=\sqrt{2} \tanh \left(\frac{\eta}{\sqrt{2}}\right)$.

It is worth mentioning here that the closed-form solution (28) has been recovered from the present Dirichlet's method that gives for $a=-1, b_{0}=1$ and $n=1$ which complements our confidence on the method. Compared to available numerical method for its solution, the Dirichlet's series gives the most accurate solution up to required accuracy with fewer number of coefficients in the

Table 2

Comparison of skin friction coefficient $f^{\prime \prime}(0)$ obtained by the Dirichlet series (22) with that of direct numerical solution.

\begin{tabular}{rllll}
\hline \multicolumn{1}{r}{$\beta$} & \multicolumn{1}{l}{$a$} & $b_{0}$ & Dirichlet series (22) & Numerical method \\
\hline-0.5 & -1.58147 & 1.250974 & -0.370497 & -0.370399 \\
0.0 & -1.318844 & 1.142742 & -0.627504 & -0.627562 \\
0.5 & -1.135838 & 1.062777 & -0.829946 & -0.829953 \\
1.0 & -1.0 & 1.0 & -0.999999 & -1.00000 \\
1.5 & -0.894650 & 0.948719 & -1.148596 & -1.148608 \\
2.0 & -0.810312 & 0.905616 & -1.281879 & -1.281838 \\
2.5 & -0.741037 & 0.868672 & -1.403451 & -1.403451 \\
3.0 & -0.683102 & 0.836422 & -1.515839 & -1.515863 \\
4.0 & -0.5914341 & 0.782446 & -1.719713 & -1.719736 \\
5.0 & -0.5220041 & 0.7386349 & -1.902512 & -1.902537 \\
10.0 & -0.3307588 & 0.5987858 & -2.631830 & -2.635153 \\
\hline
\end{tabular}

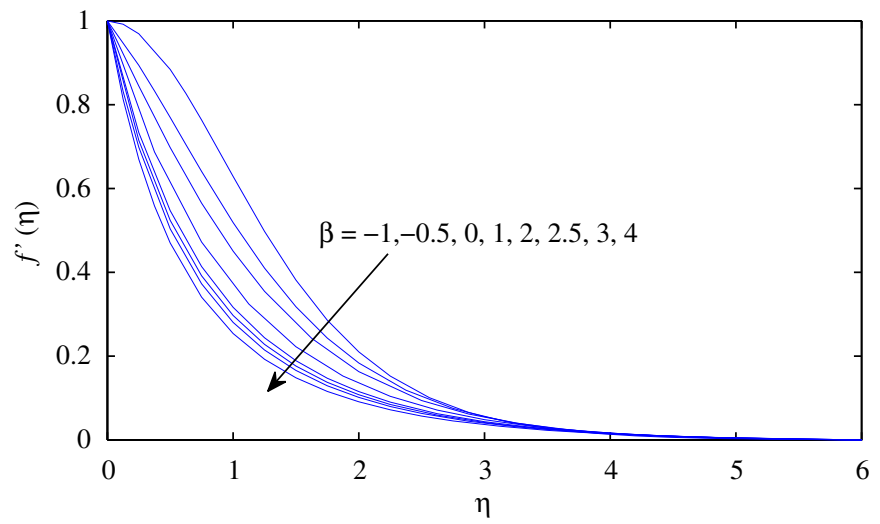

Fig. 3. Velocity profiles for the pressure gradient parameter $\beta$. 
series. Thus, this method can be extended to an ordinary differential equation of the type (20) along with the boundary conditions wherein the derivative condition at infinity should be zero.

Finally, our attention is to discuss the important results obtained in the previous two sections for different stretching parameter $\beta$ and wall stretch parameter $\epsilon$ which are given in the graphical as well as table form that provide additional insights into the problem under investigation.

\section{Results and discussion}

We have presented a new exact solution to the Falkner-Skan equation (4) for general values of pressure gradient parameter $\beta$ in the range $0<\epsilon<\frac{1}{2}$ (Section 3), and the Dirichlet series method to the boundary layer problem (20) for general values of $\beta$ for $\epsilon=0$ (Section 4). Exact solution to systems (4) and (5) has been given analytically for $0<\epsilon<\frac{1}{2}$ and numerically for $\frac{1}{2}<\epsilon<1$. Note that for $\epsilon=\frac{1}{2}$, the obvious exact solution $f(\eta)=\eta / 2$ has also been used to plot the graphs. The exhaustive analysis both analytically and numerically has been given for the Blasius equation (with $\beta=0$ ) in different frameworks, and hence no special comments are required. In order to validate our analytical results, we present our analytical results graphically in the form of velocity profiles $f^{\prime}(\eta)$ in Figs. 1(a)-(d), 2 and 3 and of the skin friction coefficient $f^{\prime \prime}(0)$ in Tables 1 and 2.

In Fig. 1(a), the velocity profiles $f^{\prime}(\eta)$ against $\eta$ are displayed for decelerated flow $\beta=-1$ for different values of $\epsilon$. It is observed from figure that the horizontal velocity profiles go asymptotically to their respective velocity ratio parameter $\epsilon$. It is also seen that as the value of $\epsilon$ increases from 0.1 to 0.5 , velocity profiles show gradual variation. In Fig. 1(b)-(d), we display the variation of velocity profiles $f^{\prime}(\eta)$ for several sets of values of accelerated flow $\beta(>0)$ and $\epsilon$. From these figures, the velocity profiles $f^{\prime}(\eta)$ satisfy the respective derivative boundary condition as $\eta \rightarrow \infty$ for all values $\beta$ and $\epsilon$ which guarantees our confidence on this new exact solution method. The Falkner-Skan equation modified by the velocity ratio parameter $\epsilon$ exhibits a very interesting velocity profiles in the boundary layer flow. Because of the last two boundary conditions in (5), the velocity profiles $f^{\prime}(\eta)$ for $\epsilon>\frac{1}{2}$ and for $\epsilon<\frac{1}{2}$ are seen to cross each other at some value of $\eta$, say $\eta_{0}$. For the Blasius flow $\beta=0$, they cross at the point $\eta_{0} \simeq 1$, whereas for accelerated flow $\beta>0, \eta_{0}<1$ always. As $\beta$ increases, the momentum boundary layer thickness decreases, physically meaning that, the effect of stretching parameter $\beta$ is to accelerate the velocity of the fluid flow which enhances the thickness of the boundary layer. This can be clearly seen from Fig. 2. This typical trend is observed for all values of $\epsilon$.

In Table 1, we compare the value of skin friction coefficient $f^{\prime \prime}(0)$ obtained by the present method (through Eqs. (10) and (14)) with the direct numerical solution of the Falkner-Skan problem for various values of $\beta$ and $\epsilon$. We see that there is an excellent agreement between two methods. Also, as expected, when $\beta$ increases, the skin friction value in absolute also increases. However, when $\epsilon$ increases for particular value of $\beta$, the skin friction coefficient decreases. Further, our computations show that when $f^{\prime \prime}(0)$ is accurate enough, the corresponding desired derived quantities can be found without much difficulty. This can be seen from the particular velocity profile in figures above.

On the other hand, once the unknown parameters $a$ and $b_{0}$ in the series (22) are calculated, we can easily compute the velocity profiles, skin friction coefficient etc. The stretching sheet problem (20) and (21) has been solved by this Dirichlet series method for all values of stretching parameter $\beta$, and thus, the results in the form of horizontal velocity profiles are presented in Fig. 3. In this case, when $\beta>0$, the surface is accelerated, $\beta=0$ implies a continuous movements of the flat surface and when $\beta<0$, the surface is decelerated. We observe from Fig. 3 that as the parameter $\beta$ increases, the momentum boundary layer thickness decreases, and eventually tends to zero as the distance increases from the initial boundary. This phenomenon can be observed for all values of $\beta$. Table 2 compares the values of $f^{\prime \prime}(0)$ obtained by the Dirichlet series method with the direct numerical solution of the problem. It is observed that results are quite remarkable. It is also observed that as $\beta$ increases, the absolute value of the boundary layer thickness also increases.

\section{Conclusions}

We have presented a new exact solution of the boundary layer flow over a semi-infinite flat plate. The flow is governed by the Falkner-Skan family of equations. The closed-form solution is obtained for $\beta=-1$ in terms of error and exponential functions which is rewritten to obtain an exact solution for all values of $\beta$. We have also presented the Dirichlet series solution for the problem in $\epsilon=0$ limit and explored that such problems can be solved without much difficulty. Results thus obtained are demonstrated in the form of velocity profiles and skin friction, and are compared with the direct numerical solution of the problem. There is a good agreement between the both solutions. It is found that the effect of pressure gradient parameter is to decrease the thickness of boundary layer. We hope to extend our present analysis by including the effect of magnetohydrodynamic boundary layer flow over a continuously stretching plate which is essentially governed by the Falkner-Skan family of equations with more general boundary conditions.

\section{Acknowledgments}

Author would like to thank Bangalore University for the financial support to carry out this research work under 'BUIRF' (Bangalore University Internal Research Funding). Author wishes to thank referees for suggestions which led to the improvement of this paper.

\section{References}

[1] H. Weyl, On the differential equations of the simplest boundary layer problems, Annals of Mathematics 43 (1942) 385-407.

[2] W.A. Coppel, On a differential equation of boundary layer theory, Philosophical Transactions of the Royal Society A 253 (1960) 101-136.

[3] D.R. Hartree, On an equation occurring in Falkner-Skan approximate treatment of the equations of the boundary layer, Proceedings of the Cambridge Philosophical Society 33 (1937) 223-239.

[4] T. Cebeci, H.B. Keller, Shooting and parallel shooting methods for solving the Falkner-Skan boundary-layer equation, Journal of Computational Physics 7 (1971) 289-300.

[5] A. Asaithambi, Numerical solution of the Falkner-Skan equation using piecewise linear functions, Applied Mathematics and Computation 159 (2004) 267-273.

[6] K.R. Rajagopal, A.S. Gupta, T.Y. Na, A note on the Falkner-Skan flows of a nonnewtonian fluid, International Journal of Non-linear Mechanics 18 (1983) 313-320.

[7] D.O. Olagunju, The Falkner-Skan flow of a viscoelastic fluid, International Journal of Non-linear Mechanics 41 (2006) 825-829.

[8] M. Anabtawi, S. Khuri, On the generalized Falkner-Skan equation governing boundary layer flow of a FENE-P fluid, Applied Mathematics Letters 20 (2007) 1211-1215.

[9] N.A. Yacob, A. Ishak, R. Nazar, I. Pop, Falkner-Skan problem for a static and moving wedge with prescribed surface heat flux in a nanofluid, International Communications in Heat and Mass Transfer 38 (2011) 149-153.

[10] N. Riley, P.D. Weidman, Multiple solutions of the Falkner-Skan equation for flow past a stretching boundary, SIAM Journal of Applied Mathematics 49 (1989) 1350-1358.

[11] H.T. Yang, L.C. Chien, Analytic solution of the Falkner-Skan equation when $\beta=-1$ and $\gamma=0$, SIAM Journal of Applied Mathematics 29 (1975) 558-569. 
[12] S.J. Liao, A uniformly valid analytic solution of two-dimensional viscous flow over a semi-infinite flat plate, The Journal of Fluid Mechanics 385 (1999) 101-128.

[13] T. Fang, J. Zhang, An exact analytical solution of the Falkner-Skan equation with mass transfer and wall stretching, International Journal of Non-Linear Mechanics 43 (2008) 1000-1006.

[14] P.L. Sachdev, R.B. Kudenatti, N.M. Bujurke, Exact analytic solution of a boundary value problem for the Falkner-Skan equation, Studies in Applied Mathematics 120 (2008) 1-16.

[15] R.B. Kudenatti, V.B. Awati, Solution of pressure gradient stretching plate with suction, Applied Mathematics and Computation 210 (2009) 151-157.

[16] N. Afzal, Momentum transfer on power law stretching plate with free stream pressure gradient, International Journal of Engineering Science 41 (2003) 1197-1207.

[17] B.C. Sakiadis, Boundary layer behavior on continuous solid surfaces: the boundary layer on a continuous flat surface, AIChE Journal 7 (1961) 221-225.
[18] L.J. Crane, Heat transfer on continuous solid surfaces, Ingenieur Archiv 43 (1974) 203-214.

[19] M.B. Zaturska, W.H.H. Banks, On the spatial stability of free convection flows in saturated porous medium, Journal of Engineering Mathematics 21 (1987) 41-46.

[20] M. Guedda, Similarity solutions of differential equations for boundary layer approximations in porous media, Zeitschrift für Angewandte Mathematik und Physik 56 (2005) 749-762.

[21] R.C. Bataller, Radiation effects for the Blasius and Sakiadis flows with a convective surface boundary condition, Applied Mathematics and Computation 206 (2008) 832-840.

[22] G. Bognar, Analytic solutions to the boundary layer problem over a stretching wall, Computers and Mathematics with Applications 61 (2011) 2256-2261.

[23] T.K. Kravnchenko, A.I. Yablonskii, Solution of an infinite boundary value problem for third order equation, Differentialnye Uraneniya 1 (1965) $327-331$.

[24] S. Riesz, Introduction to Dirichlet Series, CUP, 1957. 